\title{
Low-Grade Appendiceal Mucinous Neoplasm Presenting as a Volvulus of the Cecum: A Rare Presentation of a Rare Disease
}

ISSN: 2639-0531

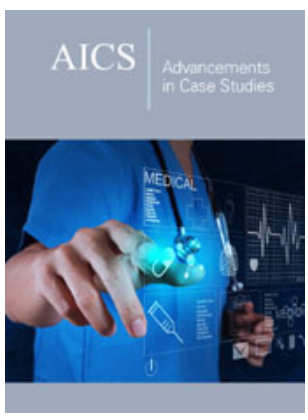

*Corresponding author: Haoran Peng, Gwinnett Medical Center, USA

Submission: 眥 September 10, 2019

Published: 海 September 19, 2019

Volume 2 - Issue 1

How to cite this article: Peng $\mathrm{H}, \mathrm{Pau}$ T, Lim T, Eichel Y, Patel N. Low-Grade Appendiceal Mucinous Neoplasm Presenting as a Volvulus of the Cecum: A Rare Presentation of a Rare Disease. Adv Case Stud.2(1). AICS.000530.2019.

DOI: 10.31031/AICS.2018.02.000530

Copyright@ Haoran Peng, This article is distributed under the terms of the Creative Commons Attribution 4.0 International License, which permits unrestricted use and redistribution provided that the original author and source are credited.

\author{
Peng $\mathrm{H}^{1}$, Pau $\mathrm{T}^{2}$, Lim $\mathrm{T}^{1}$, Eichel $\mathrm{Y}^{3}$ and Patel $\mathrm{N}^{1}$ \\ ${ }^{1}$ Internal Medicine, Gwinnett Medical Center, USA \\ ${ }^{2}$ Bostock Family Medicine, Gwinnett Medical Center, USA \\ ${ }^{3}$ Pathology, Gwinnett Medical Center, USA
}

\begin{abstract}
Low-grade appendiceal mucinous neoplasm is a rare entity that commonly presents with appendicitis-like symptoms. Cecal volvulus is a rare complication of this disease, but often requires urgent surgical intervention due to high ischemia rate. Here, we present a case of cecal volvulus caused by appendiceal mucinous neoplasm which represents an uncommon clinical finding of a rare entity.
\end{abstract}

Keywords: Appendiceal mucinous neoplasm; Appendiceal tumor; Cecal volvulus; Large-bowel obstruction

Abbreviations: LAMN: Low-Grade Appendiceal Mucinous Neoplasm; CT: Computer Tomography

\section{Introduction}

Low-grade appendiceal mucinous neoplasm (LAMN) is a rare disease with an estimated incidence of $0.3 \%$ of appendectomy specimens, commonly presents similarly to acute appendicitis [1]. Volvulus caused by LAMN is a potential but rare complication. It occurs when there is a lack of peritoneal fixation of the cecum, allowing it to be more mobile and resulting in the obstruction. However, in general, the most common portion of colon to have volvulus is the sigmoid colon, cecal volvulus occurs less frequently [2]. Here we present a case of appendiceal mucinous neoplasm causing volvulus of the cecum.

\section{Case Presentation}

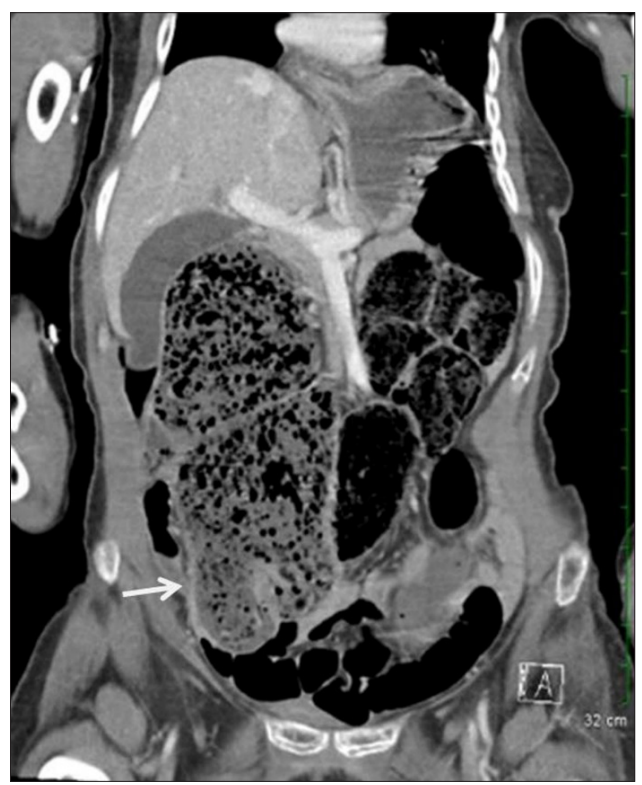

Figure 1: CT abdomen and pelvis showed a dilated cecum with large stool burden (arrow). 
A 67-year-old nonverbal female patient with severe intellectual disability, history of hysterectomy, presented to the emergency department with a 1-day history of intractable nausea and vomiting. Most of the history was obtained by her caregiver from personal home facility. The patient had multiple episodes of nonbloody emesis after breakfast the day prior to admission. She did have two normal bowel movements prior to the hospital visit on the day admission. Physical exam was significant for diffuse abdominal distention, and mild tenderness to palpation with no rebound tenderness or guarding. Lab data revealed white blood count of $9700 \mathrm{u} / \mathrm{L}$ (normal range: $5000-11000 \mathrm{u} / \mathrm{L}$ ) with $22 \%$ band neutrophil (normal range: $0-8 \%$ ), lactic acid of $2.2 \mathrm{mmol} / \mathrm{L}$ (normal range: $0.5-2.0 \mathrm{mmol} / \mathrm{L}$ ). Computer tomography (CT) scan of abdomen and pelvis revealed a markedly dilated cecum and ascending colon with large fecal burden (Figure 1).

The patient was initially treated with intravenous fluids, nasogastric tube with suction, and nothing by mouth. Surgical consultation was obtained and given the patient's clinical stability and stool burden on imaging with no obvious transition point, the surgeon believed the presentation to favor fecal impaction over cecal volvulus. Continued conservative management with enema was recommended. Patient had a large bowel movement after receiving the enema, however her distention and abdominal pain did not improve. Barium enema was then performed and confirmed cecal volvulus (Figure 2).

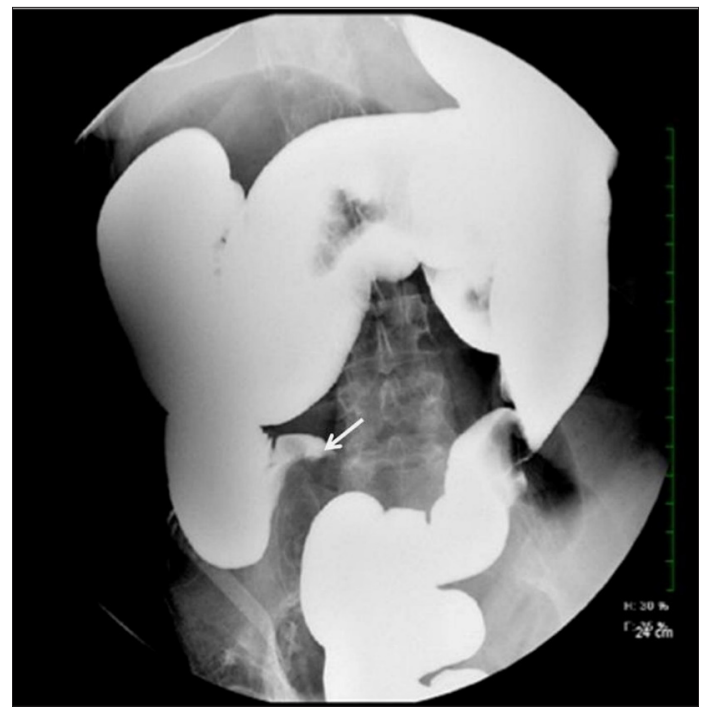

Figure 2: Barium enema film. Despite repositioning patient multiple times and waiting, no contrast was passing. "Bird's peak" appearance is compatible with cecal volvulus (arrow).

An urgent exploratory laparotomy was performed and the NG tube accidentally in day 2 post-operation, and developed revealed multiple adhesive bands and a dilated cecum with torsion hypoxia. Aspiration pneumonia was considered, and she was intraoperatively. Right hemicolectomy was performed thereafter. treated with piperacillin-tazobactam. The NG tube was replaced, The pathologic report showed a low-grade appendiceal mucinous and the ileus resolved in day 6 post-operation. She discharged neoplasm that focally invades the muscularis propria, uninvolved back to the personal care home facility after completing 7 days of margins, no high-grade cytologic features, 0/10 lymph nodes antibiotics. She followed up with General surgery and Oncology in involved (Figure 3). Patient developed postoperative ileus; the NG the outpatient clinic. No chemotherapy was planned due to early tube was kept in place for decompression. However, she pulled out stage of the disease and there was plan for active observation.

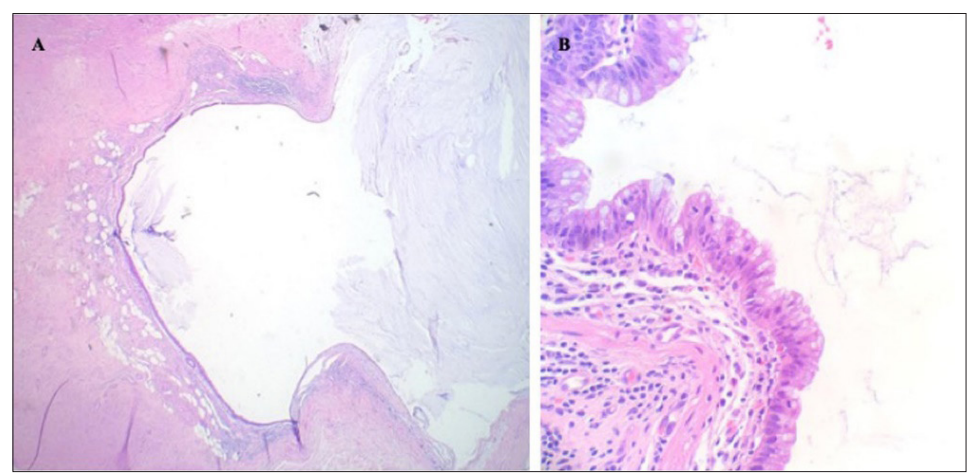

Figure 3: Histopathologic slides showed low-grade appendiceal mucinous neoplasm (A). The higher power view showed a single layer of epithelium with focal tufts, but no high-grade cytologic features or infiltrative growth pattern were identified (B). 


\section{Discussion}

Appendiceal mucinous neoplasm is a rare entity with an estimated incidence of $0.15-0.9$ per 100,000 people and represents $<1 \%$ of all appendectomy specimens [1,3]. The frequency is higher in women, with a ratio of 4:1 between women and men [4]. The 2010 WHO classification has divided appendiceal mucinous neoplasm into 3 categories: mucinous adenoma, LAMN, and appendiceal adenocarcinoma [5]. LAMN is the term to describe a true neoplasm with dysplastic epithelium that produces abundant mucin and characteristically exhibits expansile growth. It can present in a variety of ways, range from appendicitis-like symptoms (i.e. fever, nausea, vomiting, right lower quadrant pain) to palpable abdominal mass. However, cecal volvulus is an uncommon presentation [6]. A CT scan of abdomen and pelvis can demonstrate the well encapsulated low attenuation in the appendix [7]. Preoperative diagnosis is uncommon, and it is often an incidental finding at the time of surgery and occasionally discovered only at pathological exam.

In our case, the appendix was not well visualized on the CT scan. It was also difficult to differentiate the dilation of the cecum was due to volvulus or fecal impaction given a large amount of fecal burden and no obvious transition point. In view of this, a barium enema was the appropriate next step. It was both therapeutic for the fecal impaction, and also identified the cecal volvulus which is an emergent condition due to potential for intestinal ischemia and infarction. The classic "bird's beak" appearance under the barium enema is suggestive of colonic volvulus. The management of cecal volvulus is either endoscopic decompression or surgical intervention. The success rate of endoscopic decompression of cecal volvulus is only $10-15 \%$ and given that cecal volvulus also has high ischemic rate, surgical intervention is often preferred [8]. In our case, considering the low endoscopic successful rate, especially with fecal impaction, an urgent exploratory laparostomy was performed by general surgery.

There is little consensus on the optimal choice of procedure (appendectomy versus right hemicolectomy) and the approach (laparoscopic versus laparotomy) in treating LAMN. The incidence of regional lymph node spread is less than $2 \%$ with localized well differential appendiceal mucinous neoplasm, therefore, simple appendectomy is sufficient for tumors exhibiting only local involvement [9]. Right hemicolectomy is considered if there is positive margin involvement after appendectomy, and for tumors involving the peri-appendiceal area, tumor size of $2 \mathrm{~cm}$ or larger, high grade histology, or for tumors that invade the muscularis propria [10]. The right hemicolectomy was performed to our patient based on the intraoperative findings. A LAMN without lymph node metastasis was confirmed by pathology.

Our patient developed post-operative ileus and aspiration pneumonia after she is pulling out the nasogastric tube accidentally. Otherwise, she recovered well from the surgery and continued outpatient clinic follow up.

\section{Conclusion}

Cecal volvulus caused by a low-grade appendiceal mucinous adenocarcinoma is a very rare clinical presentation. CT of abdomen and pelvis is the recommended study for diagnosis. But the diagnosis is made intraoperatively and on histopathological exam. Right hemicolectomy is warranted if cecum is involved.

\section{Acknowledgement}

We would also like to show our gratitude to Drs. Pau, Lim, Eichel, and Patel for sharing their pearls of wisdom with us during the course of this case report.

\section{References}

1. Fournier K, Rafeeq S, Taggart M, Kanaby P, Ning J, et al. (2017) Lowgrade appendiceal mucinous neoplasm of uncertain malignant potential (LAMN-UMP): prognostic factors and implications for treatment and follow up. Ann Surg Oncol 24(1):187-193.

2. Halabi WJ, Jafari MD, Kang CY, Nguyen VQ, Carmichael JC, et al. (2014) Colonic volvulus in the United States: trends, outcomes, and predictors of mortality. Ann Surg 259(2): 293-301.

3. Rutledge RH, Alexander JW (1992) Primary appendiceal malignancies: rare but important. Surgery 111(3): 244-250.

4. Barlett C, Manoharan M, Jackson A (2007) Mucocele of the appendix-a diagnostic dilemma: a case report. J Med Case Rep 1: 183.

5. Tirumani SH, Fraser-Hill M, Auer R, Shabana W, Walsh C, et al. (2013) Mucinous neoplasms of the appendix: a current comprehensive clinicopathologic and imaging review. Cancer Imaging 13: 14-25.

6. Costa V, DeMuro JP (2013) Low-grade appendiceal neoplasm presenting as a volvulus of the cecum. Gastroenterol Rep (Oxf) 1(3): 207-210.

7. Kim SH, Lim HK, Lee WJ, Lim JH, Byun JY (1998) Mucocele of the appendix: Ultrasonographic and CT findings. Abdom Imaging 23(3): 292-296.

8. Perrot L, Fohlen A, Alves A, Lubrano J (2016) Management of the colonic volvulus in 2016. J Visc Surg 153(3): 183-192.

9. Yantiss RK, Shia J, Klimstra DS, Hahn HP, Odze RD, et al. (2009) Prognostic significance of localized extra-appendiceal mucin deposition in appendiceal mucinous neoplasms. Am J Surg Pathol 33(2): 248-255.

10. Gonzalez-Moreno S, Sugarbaker PH (2004) Right hemicolectomy does not confer a survival advantage in patients with mucinous carcinoma of the appendix and peritoneal seeding. Br J Surg 91(3): 304-311. 